\title{
Absence of magnetic phase separation in MnSi under pressure
}

\author{
D. Andreica, ${ }^{1,2}$ P. Dalmas de Réotier, ${ }^{3}$ A. Yaouanc, ${ }^{3}$ A. Amato, ${ }^{1}$ and G. Lapertot ${ }^{3}$ \\ ${ }^{1}$ Laboratory for Muon-Spin Spectroscopy, Paul Scherrer Institute, 5232 Villigen-PSI, Switzerland \\ ${ }^{2}$ Faculty of Physics, Babes-Bolyai University, 400084 Cluj-Napoca, Romania \\ ${ }^{3}$ CEA/DSM/Institut Nanosciences et Cryogénie, 38054 Grenoble, France
}

(Received 5 November 2009; revised manuscript received 6 January 2010; published 23 February 2010)

\begin{abstract}
We report muon spin spectroscopy data $(\mu \mathrm{SR})$ obtained under hydrostatic pressure on a large single crystal of the itinerant helimagnet $\mathrm{MnSi}$ and recorded down to $0.235 \mathrm{~K}$ and up to $15.1 \mathrm{kbar}$. Up to the critical pressure $p_{c}=14.9$ (2) kbar, where the magnetic order is suppressed, the $\mu \mathrm{SR}$ data unambiguously demonstrate that the ground state of the system is magnetic with no indication of any phase separation.
\end{abstract}

DOI: 10.1103/PhysRevB.81.060412

PACS number(s): 75.30.-m, 71.20.Lp, 76.75.+i

The strongly correlated intermetallic cubic compound $\mathrm{MnSi}$ has been known for a long time to order magnetically below $T_{c} \simeq 29 \mathrm{~K}$ in a long period helical structure. ${ }^{1,2}$ The first-order nature of the transition has only been demonstrated quite recently. ${ }^{3,4}$ The study of the effect of pressure on the magnetic phase has shown that the transition temperature decreases with pressure, remaining first order, and tending to zero at $p_{c} \simeq 14$ kbar. ${ }^{5,6}$

It is well known that phase separation might occur near the temperature of a first-order-phase transition. Previous muon spin rotation and relaxation $(\mu \mathrm{SR})$ experiments by Uemura et al. indicated a magnetic phase separation (MPS) in $\mathrm{MnSi}^{7}$ Prior to the $\mu \mathrm{SR}$ work, a nuclear magnetic resonance (NMR) study suggested MPS in the same pressure range $\Delta p \simeq 3$ kbar just below $p_{c}{ }^{8}$ However, since the NMR sample had to be powderized, strains problems could induce extrinsic MPS. Hence, probing a single crystal, as it has been done in Ref. 7, is certainly an advantage. However, the validity of the conclusions drawn from these measurements can be questioned since, according to Fig. $2 \mathrm{c}$ of Ref. 7 , solely one spontaneous frequency was detected below $T_{c}$ rather than two, as expected from previous ambient pressure measurements. ${ }^{9}$ In addition, the measurements were performed only down to $2.5 \mathrm{~K}$, which might be an issue when studying the behavior of the system near $p_{c}$ where $T_{c}$ goes to 0 .

To clarify such issues, we have carried out a series of accurate $\mu \mathrm{SR}$ experiments under pressure down to $0.235 \mathrm{~K}$ on a large MnSi single crystal. The main result of our study is that, up to $p_{c}$ and at low temperatures, the full sample volume becomes magnetic. Therefore, despite the first-order nature of the phase transition that is clearly confirmed by our data, no phase separation is detected in MnSi.

Due to its unique sensitivity to slow spin fluctuations and its local probe character providing the possibility to distinguish paramagnetic and magnetically ordered volume fractions, ${ }^{10-13}$ no matter the compound chemical composition, the $\mu \mathrm{SR}$ spectroscopy is well suited to probe whether MPS is effectively an intrinsic property of MnSi. Since our main interest was on the existence of MPS, most of the $\mu \mathrm{SR}$ measurements were performed with the so-called weaktransverse-field (WTF) method, which is well adapted to determine the magnetic volume fraction. A small field, $B_{\text {ext }}=5 \mathrm{mT}$ in our case, is applied perpendicular to the muon polarization $\mathbf{S}_{\mu}$. The depolarization function for the muons stopping in the sample is then the sum of two precessing components. While the one which arises from the paramagnetic volume precesses at a frequency associated to the applied field and is weakly damped, the other one related to muons stopped in the magnetically ordered phase, precesses at a frequency corresponding to the spontaneous field, and exhibits a strong depolarization. This latter damping stems from the relatively large magnetic field distribution at the muon site inherent to an ordered magnetic state. By carefully determining the amplitude of these components, the volume ratio between the magnetic and paramagnetic phases can be accurately determined as a function of temperature and pressure. Measurements in zero applied magnetic field were also performed at different pressures.

The $\mu \mathrm{SR}$ measurements were carried out at the general purpose decay-channel (GPD) spectrometer of the Swiss Muon Source $(\mathrm{S} \mu \mathrm{S}$, Paul Scherrer Institute, Villigen, Switzerland). A detailed description of the pressure cell used for the $\mu \mathrm{SR}$ measurements is given elsewhere. ${ }^{14,15}$ Relative to previous works, some improvements were made. The pressure cell was mounted either on the sample stick of a Janis ${ }^{4} \mathrm{He}$-flow cryostat or on the cold finger of an Oxford Instrument ${ }^{3} \mathrm{He}$ cryostat. The temperature range from 40 down to $0.235 \mathrm{~K}$ could hence be covered. The extension to low temperature by more than an order of magnitude relative to previous $\mu \mathrm{SR}$ work $^{7}$ is a key ingredient to unravel the features reported in the present study. For each pressure in this study, the exact pressure applied on the sample was measured in situ at low temperature using the pressure dependence of the superconducting transition temperatures of small pieces of either In or $\mathrm{Pb}$ placed inside the pressure cell. It is worth mentioning that the pressure determination was performed at similar temperatures as the $\mu \mathrm{SR}$ measurements themselves. The pressure transmitting medium was a 1:1 mixture of n-pentane and isoamyl alcohol which is known for the excellent hydrostaticity conditions it provides in our pressure range of interest and for the absence of hysteresis effects. ${ }^{16}$

The MnSi sample was a cylinder of $7 \mathrm{~mm}$ diameter and $19 \mathrm{~mm}$ length cut from a single crystal prepared similarly as the one used in previously published ambient pressure $\mu \mathrm{SR}$ measurements, ${ }^{17}$ elastic neutron-scattering investigation under pressure ${ }^{18}$ and thermal-expansion studies ${ }^{19}$ under pressure. The single crystal was grown by the Czochralsky pulling technique from a stoichiometric melt of high-purity 


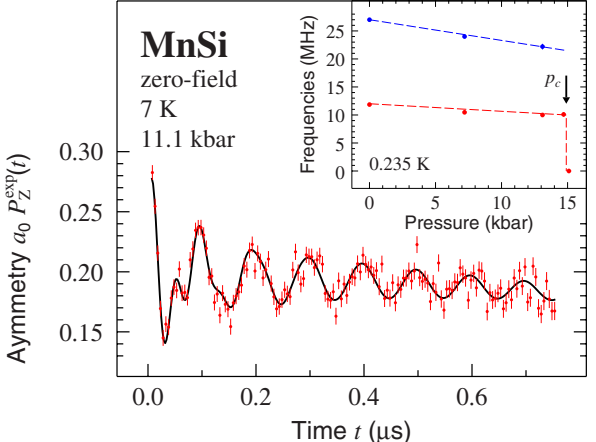

FIG. 1. (Color online) An example of a zero-field $\mu$ SR spectrum recorded on a single crystal of $\mathrm{MnSi}$ at $7 \mathrm{~K}$. The pressure as determined by the superconducting temperature of indium is $11.1 \mathrm{kbar}$. The solid line is the result of the fit consisting of two spontaneously precessing components plus an exponential spin-lattice relaxation one. The oscillations are described by exponentially damped cosine functions with frequencies $\nu_{1}=9.99(3) \mathrm{MHz}$ and $\nu_{2}=22.9(5) \mathrm{MHz}$ and an amplitude ratio similar to the one reported at ambient pressure value (Ref. 9). The amplitude of the spin-lattice relaxation channel is found to account for one third of the total $\mathrm{MnSi}$ signal, a ratio expected for a weakly anisotropic cubic magnet like $\mathrm{MnSi}$ irrespective of the orientation of the muon initial polarization relative to the crystal axes. The relaxation rate is $\lambda_{Z}=0.11(5) \mu \mathrm{s}^{-1}$. The signal from the pressure cell is characterized and described in Refs. 14 and 15. Nearly half of the measured signal originates from the sample, a much larger proportion than the $\sim 30 \%$ of the published work (Ref. 7). The insert displays the pressure dependence of the two spontaneous precession frequencies measured at $0.235 \mathrm{~K}$. At $14.7 \mathrm{kbar}$, the upper one is too heavily damped to be reliably extracted from the spectrum. The dashed lines are guides to the eyes.

elements (>99.995\%) using radio-frequency heating and a cold copper crucible. The residual resistivity ratio of such prepared crystals is about 40. Scanning electron microscope microanalysis and backscattered electron images reveal neither any deviation from the known crystal structure nor any presence of foreign phase. The possibility for offstoichiometry was carefully investigated by examining the polycrystalline ingot remaining after a pulling especially designed to consume $99 \%$ of the initial load, instead of $\simeq 25 \%$ for normal growth. No sizeable fraction of any foreign phase was found in the remaining ingot, confirming a stoichiometry in the ratio $1: 1$ in our MnSi single crystal.

In Fig. 1 a zero-field $\mu$ SR spectrum recorded at $7 \mathrm{~K}$ under $11.1 \mathrm{kbar}$ is reported. It serves to display the sample and pressure quality. The beating of the expected two oscillating components is clearly seen. Had the field distribution in the sample been large, due, for example, to a large pressure gradient or sample inhomogeneity, the higher frequency would not have been detected. Note that this higher-frequency precession was not observed in the previous $\mu$ SR work under pressure. $^{7}$ The pressure dependence of the two lowtemperature spontaneously precessing frequencies, which reflect the pressure dependence of the order parameter, is displayed in the insert of Fig. 1. An abrupt change is observed between 14.7 and $15.1 \mathrm{kbar}$, confirming the first-order nature of the phase transition. Belitz et al. predicted this type of transition to occur in weak ferromagnets. ${ }^{20}$

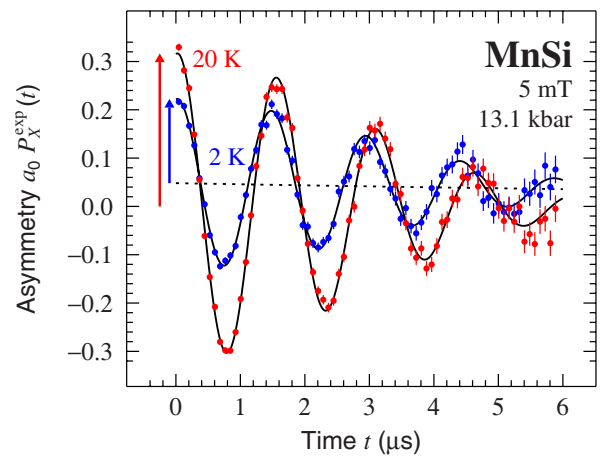

FIG. 2. (Color online) Spectra recorded in a weak transverse field of $5 \mathrm{mT}$ in MnSi under a pressure of $13.1 \mathrm{kbar}$. Note that the time scale differs completely with the one of Fig. 1. The binning of the data which is adopted here averages out the oscillations of the muon spin in the MnSi spontaneous field. The initial amplitude of the $20 \mathrm{~K}$ spectrum which is marked by the longer arrow has the expected value in our experimental conditions. At $2 \mathrm{~K}$, the precessing component observed in the plot corresponds to muons which are stopped in the pressure cell: its amplitude is indicated by the shorter arrow. The spin-lattice relaxation signature of the muons which are implanted in $\mathrm{MnSi}$ is seen as a shift in the mean value of the precessing signal. The solid lines are fits to a product of a cosine function and a Gaussian damping, with an additional exponential spin-lattice relaxation function (shown as a dotted line) for the $2 \mathrm{~K}$ spectrum. The Gaussian damping corresponds to a dominating inhomogeneous broadening.

Examples of WTF spectra are shown in Fig. 2. Muons stopped both in the pressure cell and in the MnSi crystal contribute to them. The muons implanted in paramagnetic $\mathrm{MnSi}$ and in the (non magnetic) pressure cell precess at a frequency which differs from $\gamma_{\mu} B_{\text {ext }} /(2 \pi)$ only by a small Knight shift; $\gamma_{\mu}=851.6 \mathrm{Mrad}^{-1} \mathrm{~s}^{-1} . \mathrm{T}^{-1}$ is the muon gyromagnetic ratio. Muons stopped in magnetically ordered MnSi precess at a much higher frequency (see Fig. 1 and note the time scale). Since at low temperature and room pressure it is known that the whole volume of MnSi is magnetically ordered, it is simple matter to derive the pressure cell contribution to the signal. It is then possible to determine the MnSi magnetic volume fraction. The results are shown in Fig. 3 as a function of temperature and pressure. The most obvious feature is that the whole sample volume is magnetically ordered at low temperature for all the applied pressures. The magnetic volume fraction is fitted to the phenomenological law $\left\{1+\exp \left[\left(T-T_{c}\right) / w\right]\right\}^{-1}$. With this formula, for a given pressure, $T_{c}$ is the temperature at which half of the sample volume is magnetic. The pressure dependence of $T_{c}$ will be shown and compared to the literature results in Fig. 6. Up to 13 kbar, the width of the transition observed in Fig. 3 is relatively narrow, pressure independent and about two times narrower than previously reported. ${ }^{7}$ The data taken at 14.7 kbar single out themselves: the transition width is more pronounced. This trend if confirmed in Fig. 4 by the plot of the fit parameter $w$ which is proportional to the width of the magnetic transition.

We present in Fig. 5 two zero-field spectra recorded at 14.7 and $15.1 \mathrm{kbar}$, respectively. The pressure at which the spontaneous $\mu \mathrm{SR}$ frequencies disappear from the $\mu \mathrm{SR}$ spec- 


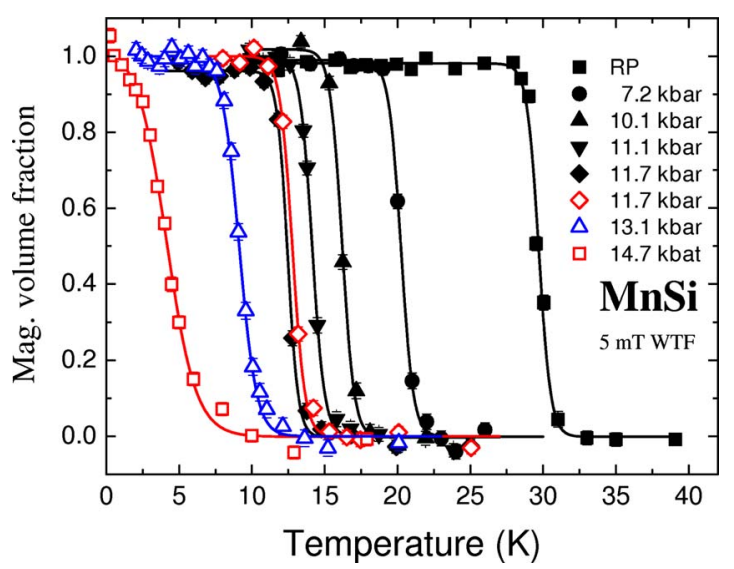

FIG. 3. (Color online) Temperature dependence of the magnetic volume fraction for a crystal of $\mathrm{MnSi}$ at different applied pressures. The data were obtained from $5 \mathrm{mT}$ transverse-field measurements. The solid lines are fits to the phenomenological law given in the main text. RP stands for room pressure.

tra at low temperatures is taken as the critical pressure $p_{c}$, where the magnetic ground state is suppressed.

The pressure dependence of the low-temperature magnetic volume fraction derived from our measurements as well as of the critical temperature are plotted in Fig. 6. The pressure dependence of the critical temperature is consistent with recently published results obtained by various techniques (thermal expansion, specific heat and ac susceptibility) up to about $13 \mathrm{kbar}$. The possible discrepancy at higher pressure (although the experimental points are somewhat distributed) may be due to the effect of different pressure media. ${ }^{19}$

It may be that the sample and pressure quality plays a role in the appearance of MPS. A very clear example is given by the research on $\mathrm{URu}_{2} \mathrm{Si}_{2}$; see Ref. 24 and references therein. Better sample and pressure quality results in a sharper transition in $\mathrm{URu}_{2} \mathrm{Si}_{2}$; i.e., no MPS is observed. Finally we interpret the data recorded in the vicinity of $p_{c}$.

We first estimate a bound on the characteristic fluctuation time for the spin dynamics under $14.7 \mathrm{kbar}$ at $0.235 \mathrm{~K}$. Since we clearly observe oscillations up to $\sim 0.2 \mu$ s, see Fig. 5, the two spontaneous fields do not flip in this time range..$^{25}$

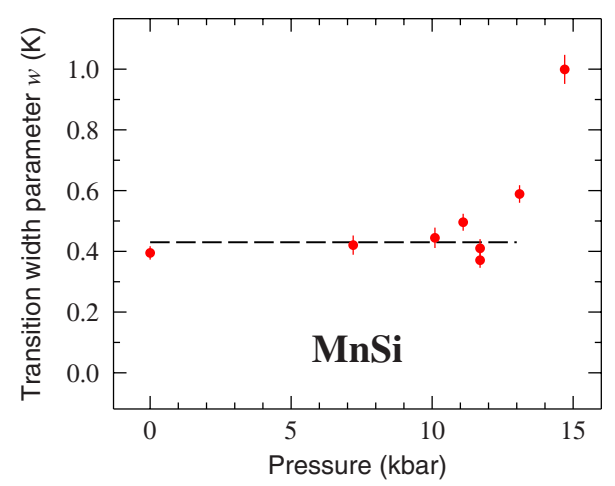

FIG. 4. (Color online) Pressure dependence of the $w$ parameter determined from the fit of the data in Fig. 3. As shown by the dashed line, $w$ is merely unchanged up to about $13 \mathrm{kbar}$.

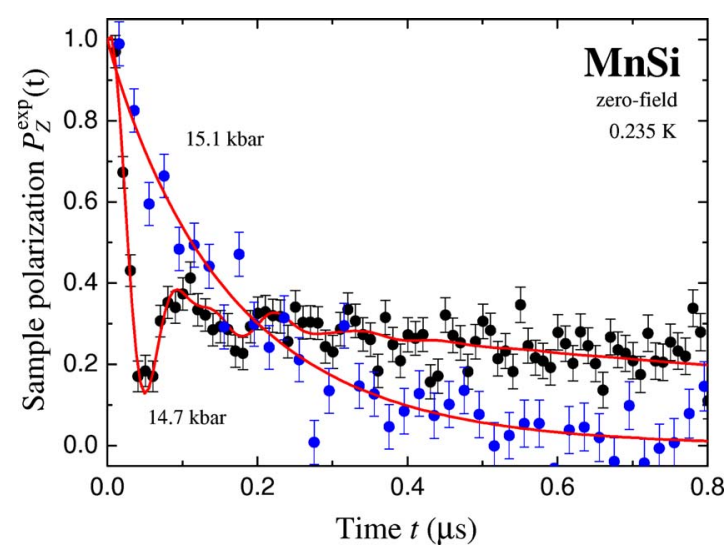

FIG. 5. (Color online) Time dependence of the zero-field muon polarization for $\mathrm{MnSi}$ recorded at $0.235 \mathrm{~K}$ on each side of the critical pressure, after subtraction of the $\mu \mathrm{SR}$ signal arising from the pressure cell. The solid lines represent the best fits to the spectra. The sample depolarization function has an exponential character at $15.1 \mathrm{kbar}$. At $14.7 \mathrm{kbar}$ it is the sum of a relaxing and of two damped oscillating signals.

Therefore the time characterizing the fluctuations of the two spontaneous fields is at least as long as $0.2 \mu \mathrm{s}$.

We now discuss the pressure inhomogeneity in our measurements. It seems that a pressure distribution of $0.5 \mathrm{kbar}$ is a reasonable value. ${ }^{19,26}$ Because the depolarization function measured at $0.235 \mathrm{~K}$ under $15.1 \mathrm{kbar}$ is fully characteristic of a paramagnetic state and the one recorded under $14.7 \mathrm{kbar}$ at the same temperature reflects a magnetic state, we infer $p_{c}=14.9$ (2) kbar. Since we now have some insight on the quality of the applied pressure, we can interpret the anomalous large width of the temperature dependence of the magnetic volume fraction observed at 14.7 kbar; see Fig. 3. We expect the $\mu \mathrm{SR}$ spectra to depend strongly on the pressure near $p_{c}$. In fact, we note that the width at $14.7 \mathrm{kbar}$ in our

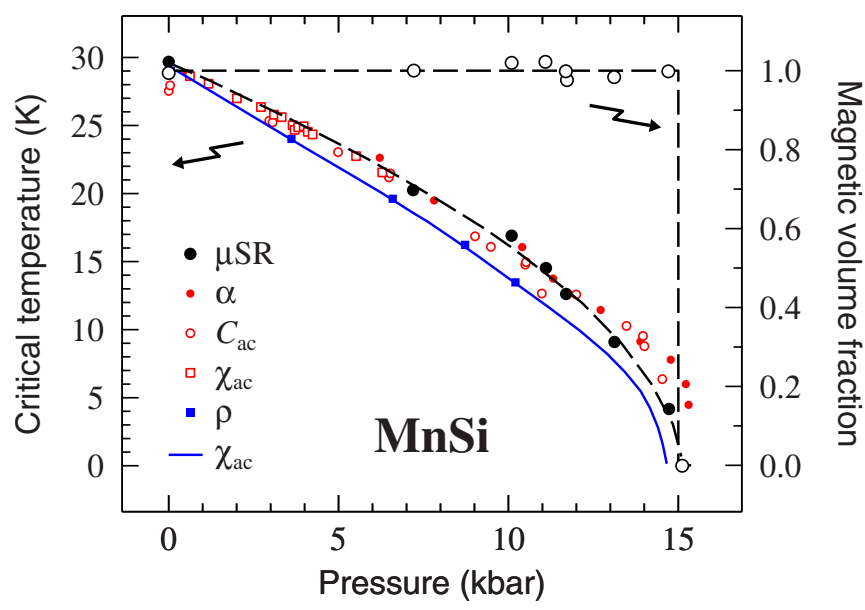

FIG. 6. (Color online) Low-temperature magnetic volume fraction and magnetic critical temperature as a function of the applied pressure determined from our $\mu \mathrm{SR}$ study. The dashed lines are guide to the eyes. The data of the pressure dependence of the critical temperature are from thermal expansion $(\alpha)$ (Ref. 19), ac specific heat $\left(C_{\mathrm{ac}}\right)$ (Ref. 19), ac susceptibility $\left(\chi_{\mathrm{ac}}\right)$ (Refs. 21 and 22), and resistivity $(\rho)$ (Ref. 23). 
case has about the value found by Uemura et $a .^{7}$ at low pressure for which they did not observe MPS.

In conclusion, our work shows the importance of pressure homogeneity and access to low temperatures for getting reliable information on the phase-separation problem. We have shown that no MPS is present in MnSi at low temperature. Our data are consistent with the existence at low temperature of a sharp transition at the critical pressure $p_{c}=14.9$ (2) kbar. Pfleiderer et al. (see also Ref. 18) recently argued for a partial magnetic order above $p_{c} \cdot{ }^{27}$ We expect that $\mu \mathrm{SR}$ should be able to study its dynamics.
We acknowledge useful conversations with B. Fåk. This research project has been partially supported by the European Commission under the 6th Framework Programme through the Key Action: Strengthening the European Research Area, Research Infrastructures (Contract No. RII3CT-2003-505925). D.A. acknowledges financial support from the Romanian CNCSIS Project No. 444/2009. Three of us (P.D.R., A.Y., and A.A.) were partially supported by the "Programme d'action integrée PAI franco-suisse Germaine de Staël." Part of this work was performed at the Swiss Muon Source, Paul Scherrer Institute, Villigen, Switzerland.
${ }^{1}$ H. J. Williams, J. H. Wernick, R. C. Sherwood, and G. K. Wertheim, J. Appl. Phys. 37, 1256 (1966).

${ }^{2}$ Y. Ishikawa, K. Tajima, D. Bloch, and M. Roth, Solid State Commun. 19, 525 (1976).

${ }^{3}$ S. M. Stishov, A. E. Petrova, S. Khasanov, G. K. Panova, A. A. Shikov, J. C. Lashley, D. Wu, and T. A. Lograsso, J. Phys.: Condens. Matter 20, 235222 (2008).

${ }^{4}$ A. E. Petrova and S. M. Stishov, J. Phys.: Condens. Matter 21, 196001 (2009).

${ }^{5}$ J. D. Thompson, Z. Fisk, and G. G. Lonzarich, Physica B 161, 317 (1989).

${ }^{6}$ A. E. Petrova, V. N. Krasnorussky, T. A. Lograsso, and S. M. Stishov, Phys. Rev. B 79, 100401(R) (2009).

${ }^{7}$ Y. J. Uemura et al., Nat. Phys. 3, 29 (2007).

${ }^{8}$ W. Yu, F. Zamborszky, J. D. Thompson, J. L. Sarrao, M. E. Torelli, Z. Fisk, and S. E. Brown, Phys. Rev. Lett. 92, 086403 (2004).

${ }^{9}$ R. Kadono, T. Matsuzaki, T. Yamazaki, S. R. Kreitzman, and J. H. Brewer, Phys. Rev. B 42, 6515 (1990).

${ }^{10}$ P. Dalmas de Réotier and A. Yaouanc, J. Phys.: Condens. Matter 9, 9113 (1997).

${ }^{11}$ A. Amato, Rev. Mod. Phys. 69, 1119 (1997).

${ }^{12}$ P. Dalmas de Réotier, P. C. M. Gubbens, and A. Yaouanc, J. Phys.: Condens. Matter 16, S4687 (2004).

${ }^{13}$ A. Amato, M. J. Graf, A. de Visser, H. Amitsuka, D. Andreica, and A. Schenck, J. Phys.: Condens. Matter 16, S4403 (2004).

${ }^{14}$ D. Andreica, Ph.D. thesis, ETH, Zürich, 2001, Chap. 5.
${ }^{15}$ S. Sakarya et al., Phys. Rev. B 81, 024429 (2010).

${ }^{16}$ N. P. Butch, J. R. Jeffries, D. A. Zocco, and M. B. Maple, High Press. Res. 29, 335 (2009).

${ }^{17}$ A. Yaouanc, P. Dalmas de Réotier, P. C. M. Gubbens, S. Sakarya, G. Lapertot, A. D. Hillier, and P. King, J. Phys.: Condens. Matter 17, L129 (2005).

${ }^{18}$ B. Fåk, R. A. Sadykov, J. Flouquet, and G. Lapertot, J. Phys.: Condens. Matter 17, 1635 (2005).

${ }^{19}$ A. Miyake, A. Villaume, Y. Haga, G. Knebel, B. Salce, G. Lapertot, and J. Flouquet, J. Phys. Soc. Jpn. 78, 044703 (2009).

${ }^{20}$ D. Belitz, T. R. Kirkpatrick, and T. Vojta, Phys. Rev. Lett. 82, 4707 (1999).

${ }^{21}$ C. Pfleiderer, G. J. McMullan, S. R. Julian, and G. G. Lonzarich, Phys. Rev. B 55, 8330 (1997).

${ }^{22}$ A. E. Petrova, V. N. Krasnorussky, J. Sarrao, and S. M. Stishov, Phys. Rev. B 73, 052409 (2006).

${ }^{23}$ C. Thessieu, J. Flouquet, G. Lapertot, A. N. Stepanov, and D. Jaccard, Solid State Commun. 95, 707 (1995).

${ }^{24}$ H. Amitsuka, K. Matsuda, I. Kawasaki, K. Tenya, M. Yokoyama, C. Sekine, N. Tateiwa, T. C. Kobayashi, S. Kawarazaki, and H. Yoshizawa, J. Magn. Magn. Mater. 310, 214 (2007).

${ }^{25}$ P. Dalmas de Réotier et al., Phys. Rev. Lett. 96, 127202 (2006).

${ }^{26}$ A. S. Rüetschi and D. Jaccard, Rev. Sci. Instrum. 78, 123901 (2007).

${ }^{27}$ C. Pfleiderer, D. Reznik, L. Pintschovius, H. v. Löhneysen, M. Garst, and A. Rosch, Nature (London) 427, 227 (2004). 